\title{
Quasiparticle properties of a coupled quantum-wire electron-phonon system
}

\author{
Hwang, E. H.; Hu, Ben Yu-Kuang; Sarma, S. Das
}

Published in:

Physical Review B

Link to article, DOI:

10.1103/PhysRevB.54.4996

Publication date:

1996

Document Version

Publisher's PDF, also known as Version of record

Link back to DTU Orbit

Citation (APA):

Hwang, E. H., Hu, B. Y-K., \& Sarma, S. D. (1996). Quasiparticle properties of a coupled quantum-wire electronphonon system. Physical Review B, 54(7), 4996-5005. https://doi.org/10.1103/PhysRevB.54.4996

\section{General rights}

Copyright and moral rights for the publications made accessible in the public portal are retained by the authors and/or other copyright owners and it is a condition of accessing publications that users recognise and abide by the legal requirements associated with these rights.

- Users may download and print one copy of any publication from the public portal for the purpose of private study or research.

- You may not further distribute the material or use it for any profit-making activity or commercial gain

- You may freely distribute the URL identifying the publication in the public portal 


\title{
Quasiparticle properties of a coupled quantum-wire electron-phonon system
}

\author{
E. H. Hwang \\ Department of Physics, University of Maryland, College Park, Maryland 20742-4111 \\ Ben Yu-Kuang $\mathrm{Hu}$ \\ Department of Physics, University of Maryland, College Park, Maryland 20742-4111 \\ and Mikroelektronik Centret, Danmarks Tekniske Universitet, Bygning 345ф, DK-2800 Lyngby, Denmark \\ S. Das Sarma \\ Department of Physics, University of Maryland, College Park, Maryland 20742-4111
}

(Received 5 February 1996)

\begin{abstract}
We study leading-order many-body effects of longitudinal-optical phonons on electronic properties of onedimensional quantum-wire systems. We calculate the quasiparticle properties of a weakly polar onedimensional electron gas in the presence of both electron-phonon and electron-electron interactions. The leading-order dynamical screening approximation ( $G W$ approximation) is used to obtain the electron selfenergy, the quasiparticle spectral function, and the quasiparticle damping rate in our calculation by treating electrons and phonons on an equal footing. Our theory includes effects (within the random-phase approximation) of Fermi statistics, Landau damping, plasmon-phonon mode coupling, phonon renormalization, dynamical screening, and impurity scattering. In general, electron-electron and electron-phonon many-body renormalization effects are found to be nonmultiplicative and nonadditive in our theoretical results for quasiparticle properties. [S0163-1829(96)05032-1]
\end{abstract}

\section{INTRODUCTION}

There has been a great deal of recent interest in ultranarrow confined semiconductor systems, called quantum-wire structures, where the motion of the electrons is essentially restricted to be one-dimensional (1D) because of their potential, both, in basic physics and for applications to different device concepts. ${ }^{1}$ Thus, both from the fundamental and applied physics viewpoints, there is interest in understanding the electronic properties of quasi-one-dimensional quantum wires. The quantum-wire structures are usually fabricated by lithographic techniques, ${ }^{2}$ epitaxial growth on tilted superlattices, ${ }^{3}$ overgrowth of preetched $V$-groove patterns, ${ }^{4}$ stress patterning, ${ }^{5}$ and the cleaved edge overgrowth method, ${ }^{6}$ which has been used to produce narrow quantum wires with atomic scale definition.

In a doped polar semiconductor (in which most of the quantum-wire structures are fabricated), free carriers couple to the longitudinal-optical (LO) phonons of the underlying lattice through the long-range polar Fröhlich coupling. The carrier-LO-phonon interaction leads to polaronic many-body renormalization of the single-particle free carrier properties, e.g., polaronic effective mass renormalization, lowering of the effective band edge, inelastic scattering, broadening of the quasiparticle spectral function, etc. Even though the weakly polar $I I I-V$ materials have rather small Fröhlich coupling constants, the electronic properties can still be substantially modified by the Fröhlich interaction, and, conversely, the phonon properties are affected by the Coulomb interaction between the electrons. Coulomb and Fröhlich interactions cannot be disentangled in experimental situations, because many-body renormalization effects due to electronelectron and electron-phonon interactions in such a coupled system are fundamentally nonmultiplicative in nature (as well as being nonadditive).

Our goal is to study a coupled 1D electron-phonon manybody system treating electrons and phonons on an equal footing. Direct interaction between electrons via the Coulomb interaction and polar electron LO-phonon interaction (which is fundamentally Coulombic in origin, arising from the dynamical interaction between charge carriers and lattice ions) via the Fröhlich coupling are among the most extensively studied many-body interactions in solid-state physics. In a two-dimensional electron gas, quasiparticle properties of a polar electron gas have been extensively studied by treating the electron-electron and electron-phonon interactions on an equal footing. ${ }^{7}$ In Ref. 7, Jalabert and Das Sarma find that the Coulomb and Fröhlich interaction effects are nonmultiplicative, and the actual many-body correction for a $2 \mathrm{D}$ electron gas is substantially different from the one-polaron result (i.e., a single electron coupled to an LO-phonon system). In spite of substantial current interest in the properties of a polar quasi-one-dimensional electron gas (1DEG) existing in GaAs quantum wires, there has been no detailed quantitative study of 1D quasiparticle properties including both the electron-electron and electron-phonon interactions. In a GaAs (or, other $I I I-V$ materials) quantum wire, the electronic energy scales (Fermi and plasmon energies) can be comparable to the LO-phonon energy, which produces strong mode coupling between plasmons and LO-phonon modes. ${ }^{8}$ Thus, a complete many-body analysis based on treating electrons, phonons, and plasmons equivalently within the same approximation scheme is needed. We provide such an analysis in this paper based on the leading-order many-body perturbation theory. The Fröhlich electron-LOphonon coupling constant in GaAs is small, making a weak- 
coupling diagrammatic expansion for calculating quasiparticle properties a meaningful approximation.

Well-established theoretical results ${ }^{9,10}$ (TomonagaLuttinger model) predict unusual non-Fermi liquidlike properties for an interacting 1D system. No matter how weak the electron-electron interaction, the noninteracting Fermi surface is nonperturbatively unstable in the presence of interaction. While the instability of a noninteracting 1D Fermi gas to an interacting 1D Tomonaga-Luttinger liquid is a wellestablished theoretical concept, the actual quantitative effect associated with the nonperturbative disappearance of the Fermi surface is, in fact, rather small at finite temperatures in a weakly interacting 1D system such as a GaAs quantumwire 1DEG. Most of the experimentally observed electronic properties of semiconductor quantum wires have so far been interpreted in the literature as effective 1D Fermi liquids, albeit at finite temperature and in the presence of impurity scattering induced collision broadening. Indeed, based on a weak-coupling diagrammatic expansion in dynamically screened Coulomb interaction, it has recently been argued ${ }^{11}$ that 1D electrons in GaAs quantum wires behave, for the most practical purposes, as a thermally broadened Fermi liquid at finite temperatures and, most importantly, in the presence of impurity scattering effects which are invariably present in real semiconductor quantum wires. The physical reason behind this result is that impurity scattering cuts off the emission of arbitrarily low-energy plasmons by overdamping them, and the instability of the Fermi surface, arising from the decay of quasiparticles into low-energy plasmons, is prevented. Our approach in this paper is to include electron-LO-phonon Fröhlich interaction in this theory by doing an expansion in leading order dynamically screened Coulomb plus Fröhlich interactions, both in the presence and absence of impurity scattering effects for the purpose of comparison. We note in this context that (1) the leadingorder calculation, ${ }^{11}$ in the absence of impurity scattering, is consistent with the nonperturbative Tomonaga-Luttinger result in the sense that it indicates a non-Fermi liquid type behavior (with different analytic properties, however); (2) the nonperturbative Tomonaga-Luttinger liquid type calculations have only been carried out for the point contact model electron-electron interaction, whereas our work uses the true long-range Coulomb (and Fröhlich) interaction as the bare interaction.

Usually the quantum-wire confinement in the $z$ direction (taken to be the growth direction) is much stronger than the confinement in the $y$ direction in real systems (assuming the 2DEG to be confined in the $x-y$ plane). The electron is typically confined in the $z$ direction on the order of less than 100 $\AA$, whereas currently the best confinement in the $y$ direction is approximately $300 \AA$, leading to at least an order of magnitude difference in the energy-level spacings of the $y$ (a few $\mathrm{meV}$ ) and the $z$ (the order of tens of meV) direction. In this paper, we therefore assume for the sake of simplicity that the electron gas has zero thickness in the $z$ direction, but has a finite width in the $y$ direction. This, in fact, is one of the common models used in studying 1D quantum-wire structures. Confinement of the electrons in the $y$ and $z$ directions leads to the quantization of energy levels into different subbands. We assume the 1D quantum limit where the energy separation between the lowest-energy (ground) and higher- (a)

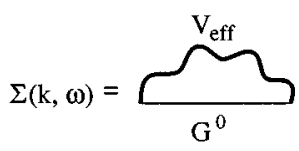

(b)

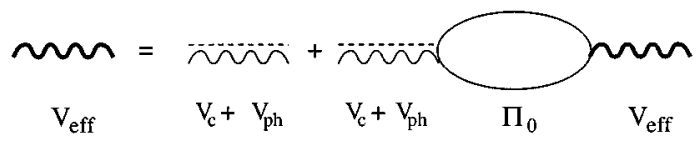

(c)

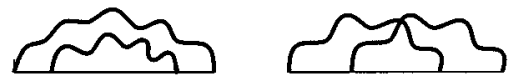

FIG. 1. (a) Electron self-energy in leading order in the effective dynamical interaction. (b) Effective dynamical interaction (thick wiggly line) $V_{\text {eff }}$ calculated in the RPA. Thin wiggly lines (dashed lines) represent the Coulomb electron-electron interaction $V_{c}$ (the LO-phonon-mediated electron-electron interaction $V_{\mathrm{ph}}$ ), and the bubble the irreducible polarizability $\Pi_{0}$. (c) Higher-order selfenergy diagrams neglected in our calculation.

energy (excited) subbands is so much larger than all other energy scales in the problem that the higher subbands can be ignored. In this paper, we calculate in the 1D quantum limit the zero temperature electron self-energy from the effective total dynamical electron-electron interaction, which is calculated by taking into account both the Coulomb electronelectron interaction and the LO-phonon-mediated Fröhlich interaction, within the random-phase approximation (RPA). We treat electron-electron and electron-phonon interactions equally, and our only approximations are the use of the RPA and the neglect of vertex corrections, i.e., we keep the lowest order diagram in the total effective dynamically screened interaction. This approximation has long been employed successfully to calculate properties of three- and twodimensional electron systems. ${ }^{12,13}$ Furthermore, the RPA gives the exact low-energy screening properties of onedimensional systems. ${ }^{14}$ The Feynman diagrams for the selfenergy and the effective interaction in these approximations are shown in Fig. 1. We ignore diagrams which are higher order in the screened interaction, such as the diagram shown in Fig. 1(c).

This paper is organized as follows. In Sec. II, we introduce our model for the coupled $1 \mathrm{D}$ electron-LO-phonon system. In Sec. III, we describe our self-energy and spectral function calculations for the quantum wire at $T=0$. In Sec. IV, we present our numerical results for various quasiparticle properties. We provide a conclusion in Sec. V. We note that the middle part of the paper (Sec. III and parts of Sec. IV) contains technical details of our theory. For readers not interested in technical details of the theory we suggest skipping Sec. III and the first half of Sec. IV and going directly to the last part of Sec. IV in which the quasiparticle damping rate is discussed. Throughout this paper, we have used the terminology in which we refer to the situation having both Coulomb and Fröhlich interactions as the coupled system, whereas the situation with only the Coulomb interaction (i.e., with the Fröhlich coupling turned off) is called the uncoupled system. Also note that in this paper, $\hbar=1$.

\section{MODEL}

Our model consists of a 1DEG coupled to bulk dispersionless LO phonons at zero temperature. Electrons interact 
among themselves through the Coulomb interaction and through virtual-LO-phonon exchange via the Fröhlich interaction. In calculating the effective $1 \mathrm{D}$ electron-phonon interaction, we sum over the phonon wave vector in the other two dimensions in the standard manner. ${ }^{7}$ The Coulomb interaction is logarithmically divergent in the $1 \mathrm{D}$ wave vector space, and, therefore, we use the more realistic finite width quantum-wire model. ${ }^{15}$ In our extreme quantum limit model where only the lowest $1 \mathrm{D}$ subband is occupied by the electrons, we obtain the Coulomb interaction matrix element $V_{c}(q)$ by taking the quantizing confinement potential to be of infinite square well type, ${ }^{11,16}$

$$
\begin{aligned}
V_{c}(q)= & \frac{2 e^{2}}{\epsilon_{\infty}} \int_{0}^{1} d x K_{0}(\text { qax })[(1-x)[2+\cos (2 \pi x)] \\
& \left.+\frac{3}{2 \pi} \sin (2 \pi x)\right],
\end{aligned}
$$

where $K_{0}$ the zeroth-order modified Bessel function of the second kind, $a$ the width of the 1DEG (i.e., the effective confinement width), and $\epsilon_{\infty}$ is the high frequency background lattice dielectric constant. In the long wavelength limit, its asymptotic form becomes

$$
V_{c}(q)=\frac{2 e^{2}}{\epsilon_{\infty}} K_{0}(q a) .
$$

The LO-phonon-mediated electron-electron interaction is dependent on wave vector and frequency,

$$
V_{\mathrm{ph}}(q, \omega)=M_{q}^{2} D_{0}(\omega) .
$$

$M_{q}$ is the effective 1D Fröhlich matrix element given by

$$
M_{q}^{2}=V_{c}(q) \frac{\omega_{\mathrm{LO}}}{2}\left[1-\frac{\epsilon_{\infty}}{\epsilon_{0}}\right],
$$

where $\omega_{\mathrm{LO}}$ is the LO-phonon frequency, $\epsilon_{0}$ is the static lattice dielectric constant. The unperturbed retarded bare LOphonon propagator is given by

$$
D_{0}(\omega)=\frac{2 \omega_{\mathrm{LO}}}{\omega^{2}-\omega_{\mathrm{LO}}^{2}+i 0^{\dagger} \operatorname{sgn}(\omega)} .
$$

The effective electron-electron interaction is obtained in the RPA by summing [Fig. 1(b)] all the bare bubble diagrams,

$$
V_{\mathrm{eff}}(q, \omega)=\frac{V_{c}(q)+V_{\mathrm{ph}}(q, \omega)}{1-\left[V_{c}(q)+V_{\mathrm{ph}}(q, \omega)\right] \Pi_{0}(q, \omega)}=\frac{V_{c}(q)}{\epsilon_{t}(q, \omega)},
$$

where $\Pi_{0}(q, \omega)$ is the complex irreducible 1D polarizability given by the bare bubble diagram. The analytic form of $\Pi_{0}(q, \omega)$ for complex frequency is given by ${ }^{15}$

$$
\Pi_{0}(q, z)=\frac{m}{\pi q} \ln \left[\frac{z^{2}-\left(q^{2} / 2 m-q v_{F}\right)^{2}}{z^{2}-\left(q^{2} / 2 m+q v_{F}\right)^{2}}\right],
$$

where the principal value of logarithm (i.e., $-\pi$ $<\operatorname{Im}[\ln (x)]<\pi)$ should be taken. In evaluating $\Pi_{0}(q, \omega)$ for real frequency, the limit $z=\omega+i 0^{\dagger}$ is taken. $\left(v_{F}\right.$ is the Fermi velocity.) $\epsilon_{t}(q, \omega)$ is the total dielectric function, which has an electron and a phonon component,

$$
\begin{aligned}
\epsilon_{\mathrm{t}}(q, \omega)= & 1-V_{c}(q) \Pi_{0}(q, \omega) \\
& +\frac{1-\epsilon_{\infty} / \epsilon_{0}}{\epsilon_{\infty} / \epsilon_{0}-\omega^{2} / \omega_{\mathrm{LO}}^{2}-i \operatorname{sgn}(\omega) 0^{\dagger}} .
\end{aligned}
$$

We include collisional broadening effects arising from electron-impurity scattering by appropriately modifying ${ }^{11}$ our dielectric function. Impurity effects in screening are usually introduced diagrammatically by including impurity ladder diagrams into the electron-hole bubble. Since the exact expression for the polarizability within this ladder vertex diagram approach is complicated, we use the particleconserving polarizability function, $\Pi_{\gamma}(q, \omega)$, given by Mermin. ${ }^{17}$ Impurity scattering self-energy effects are not included in the single-electron Green's function. In the presence of an impurity scattering induced level broadening of $\gamma$, the polarizability function is given by

$$
\Pi_{\gamma}(q, \omega)=\frac{(\omega+i \gamma) \Pi_{0}(q, \omega+i \gamma)}{\omega+i \gamma\left[\Pi_{0}(q, \omega+i \gamma) / \Pi_{0}(q, 0)\right]} .
$$

\section{ELECTRON SELF-ENERGY}

The electron Green's function $G(k, \omega)$, or equivalently, the electron self-energy, $\Sigma(k, \omega)=G_{0}^{-1}(k, \omega)-G^{-1}(k, \omega)$, where $G_{0}(k, \omega)$ is the bare noninteracting Green's function, provides a complete description of all single-electron properties of an interacting many-electron system. Knowledge of $G(k, \omega)$ or $\Sigma(k, \omega)$ allows one to calculate many experimentally observable one-electron properties of a system, such as the spectral density function, effective mass, scattering rate, lifetime, distribution function, band gap renormalization, etc. The electron self-energy of the coupled system within the leading-order [Fig. 1(a)] $G W$ approximation ${ }^{18}$ neglecting vertex corrections at $T=0$ is given by

$$
\Sigma^{t}(k, \omega)=i \int_{-\infty}^{\infty} \frac{d q}{2 \pi} \int_{-\infty}^{\infty} \frac{d \omega^{\prime}}{2 \pi} V_{\mathrm{eff}}^{t}\left(q, \omega^{\prime}\right) G_{0}^{t}\left(k-q, \omega-\omega^{\prime}\right),
$$

where $G_{0}^{t}$ is the Green's function for the noninteracting electron gas (the superscripts $t$ are to denote time ordering; otherwise, the functions in this paper are retarded),

$$
G_{0}^{t}(k, \omega)=\frac{1}{\omega-\xi(k)+i 0^{\dagger} \operatorname{sgn}\left(k-k_{F}\right)},
$$

with $\xi(k)=\left(k^{2}-k_{F}^{2}\right) / 2 m$. The time-ordered quantities are easily related to the retarded quantities. ${ }^{10}$ The self-energy can be separated into a frequency-independent exchange contribution and a correlation part,

$$
\Sigma(k, \omega)=\Sigma_{\mathrm{ex}}(k)+\Sigma_{\text {cor }}(k, \omega) .
$$

The exchange or Hartree-Fock contribution is given by

$$
\Sigma_{\mathrm{ex}}(k)=-\int \frac{d q}{2 \pi} n_{F}(k+q) V_{c}(q),
$$


where $n_{F}(k+q)=\theta\left(k_{F}-|k+q|\right)$ is the Fermi function at $T=0$, and $\Sigma_{\text {cor }}(k, \omega)$ is defined to be the part of $\Sigma(k, \omega)$ not included in $\Sigma_{\text {ex }}(k)$. In the $G W$ approximation, the $\Sigma_{\text {cor }}(k, \omega)$ can be written in the line and pole decomposition $^{10,19}$

$$
\Sigma_{\text {cor }}(k, \omega)=\Sigma_{\text {line }}(k, \omega)+\Sigma_{\text {pole }}(k, \omega),
$$

where

$$
\begin{aligned}
\Sigma_{\text {line }}(k, \omega)= & -\int_{-\infty}^{\infty} \frac{d q}{2 \pi} V_{c}(q) \int_{-\infty}^{\infty} \frac{d \omega^{\prime}}{2 \pi} \frac{1}{(\xi(k+q)-\omega)-i \omega^{\prime}} \\
& \times\left[\frac{1}{\epsilon_{t}\left(q, i \omega^{\prime}\right)}-1\right],
\end{aligned}
$$

and

$$
\begin{aligned}
\Sigma_{\text {pole }}(k, \omega)= & \int_{-\infty}^{\infty} \frac{d q}{2 \pi}\{\theta[\omega-\xi(k+q)]-\theta[-\xi(k+q)]\} \\
& \times V_{c}(q)\left[\frac{1}{\epsilon_{t}[q, \xi(k+q)-\omega]}-1\right] .
\end{aligned}
$$

The $\Sigma_{\text {line }}(k, \omega)$ is completely real because $\epsilon_{t}\left(q, i \omega^{\prime}\right)$ is real and even with respect to $\omega^{\prime}$. Thus, the imaginary part of the self-energy comes entirely from $\operatorname{Im}\left[\Sigma_{\text {pole }}\right]$. The pole contribution of the self-energy can be written as

$$
\begin{aligned}
\Sigma_{\text {pole }}(k, \omega)= & {\left[\int_{k_{F}}^{k_{0}(\omega)} \theta(\omega)-\int_{0}^{k_{F}} \theta\left(-\omega-E_{F}\right)\right.} \\
& \left.-\int_{k_{0}(\omega)}^{k_{F}} \theta\left(\omega+E_{F}\right) \theta(-\omega)\right] F(q, k, \omega) d q,
\end{aligned}
$$

where $\quad k_{0}(\omega)=\sqrt{2 m\left(\omega+E_{F}\right)} \quad$ and $\quad F(q, k, \omega)$ $=1 / 2 \pi[f(q, k, \omega)+f(-q, k, \omega)]$, where $f(q, k, \omega)$ is given by

$$
f(q, k, \omega)=V_{c}(q-k)\left[\frac{1}{\epsilon_{t}[q-k, \xi(q)-\omega]}-1\right] .
$$

Quasiparticle excitation energies $\omega_{q}$ of the system are obtained from the real part of the self-energy $\operatorname{Re}[\Sigma(k, \omega)]$ by solving Dyson's equation,

$$
\omega_{q}(k)-\xi(k)-\operatorname{Re} \Sigma(k, \omega)=0 .
$$

Once the self-energy $\Sigma(k, \omega)$ is known, the single-particle spectral function $A(k, \omega)$ is readily calculated. $A(k, \omega)$ contains an important information about the dynamical structure of the system and is given by

$$
A(k, \omega)=\frac{2|\operatorname{Im} \Sigma(k, \omega)|}{\{\omega-\xi(k)-\operatorname{Re} \Sigma(k, \omega)\}^{2}+\{\operatorname{Im} \Sigma(k, \omega)\}^{2}} .
$$

If $\operatorname{Im}[\Sigma(k, \omega)]$ goes to zero faster than $|\omega|$ as $\omega \rightarrow \omega_{q}(k)$, the spectral function becomes a $\delta$-function peak at the quasiparticle energy $\omega_{q}$,

$$
A(k, \omega)=Z(k) \delta\left[\omega-\omega_{q}(k)\right] .
$$

The strength of the peak is given by the quasiparticle renormalization factor,

$$
Z(k)=\left[1-\left.\frac{\partial \operatorname{Re} \Sigma(k, \omega)}{\partial \omega}\right|_{\omega=\omega_{q(k)}}\right]^{-1} .
$$

The characteristic of a Fermi system is that it has a finite discontinuity at the Fermi momentum in the momentum distribution function. If $Z\left(k_{F}\right) \neq 0$, the electron distribution as a function of momentum has a finite discontinuity at the Fermi momentum. Thus, if $A\left(k_{F}, \omega\right)$ shows a $\delta$-function peak at $k=k_{F}$ and $\omega=0$ the system is a Fermi liquid. However, if $\operatorname{Im}\left[\Sigma\left(k_{F}, \omega\right)\right]$ goes to zero slower than $\omega$ as $\omega \rightarrow 0$, then the spectral function $A\left(k_{F}, \omega=0\right)$ has finite broadening at $k_{F}$ and is not a $\delta$-function, implying that the system is not a Fermi liquid.

The spectral function $A(k, \omega)$ can be interpreted as a probability that an electron has a momentum $k$ and energy $\omega$, and satisfies the sum rule

$$
\int_{-\infty}^{\infty} \frac{d \omega}{2 \pi} A(k, \omega)=1
$$

which is equivalent to the conservation of the number of particles (electrons) as the interaction is turned on, starting from a noninteracting picture. In all our calculations, this sum rule is explicitly checked and is found to be satisfied to within less than a percent. The quasiparticle broadening or the damping rate $\Gamma(k)$ is given by the imaginary part of the self-energy,

$$
\Gamma(k)=-\operatorname{Im} \Sigma(k, \xi(k)),
$$

where $\xi(k)=k^{2} / 2 m-\mu$ is the quasiparticle energy measured with respect to the chemical potential $\mu$. From the damping rate, we can calculate the quasiparticle scattering rate $2 \Gamma(k)$, the inelastic lifetime $\tau(k)=[2 \Gamma(k)]^{-1}$, and the inelastic mean free path $l(k)=v(k) \tau(k)$, where $v(k)$ is the electron velocity.

\section{NUMERICAL RESULTS}

In Fig. 2 we show the real and the imaginary parts of the self-energy and spectral functions for uncoupled (thin lines) and coupled (thick lines) systems without any impurity scattering. We show the self-energies and spectral functions only at $k=0$ (band edge) and $k=k_{F}$ (Fermi energy). We use $E_{F}$ $\left(k_{F}\right)$ as our unit of energy (momentum) throughout our discussions. In this calculation, we use the parameters corresponding to GaAs: $m=0.07 m_{e},\left(m_{e}\right.$ is the free electron mass), $\epsilon_{0}=12.9, \epsilon_{\infty}=10.9$, and $\omega_{\mathrm{LO}}=36.8 \mathrm{meV}$. The well width of $a=100 \AA$ and the electron gas density of $n=0.56 \times 10^{6} \mathrm{~cm}^{-1}$, which corresponds to a Fermi energy $E_{F} \approx 4.4 \mathrm{meV}$ and a dimensionless density parameter $r_{\mathrm{s}}=4 m e^{2} / \pi k_{F} \epsilon_{0}=1.4$ with $k_{F}=\pi n / 2$, are used for this calculation. The intersections of $\operatorname{Re}[\Sigma(k, \omega)]$ and the straight line $\omega-\xi(k)-\mu$ indicate the solutions to Dyson's equation. For $k=0$ and the uncoupled system, we find three solutions of the Dyson's equation [Eq. (19)]. Two solutions (the first and the third one from $\omega=0$ ) show the $\delta$-function peaks in the spectral function, but the second solution has a broad incoherent structure indicating finite damping in the spectral 

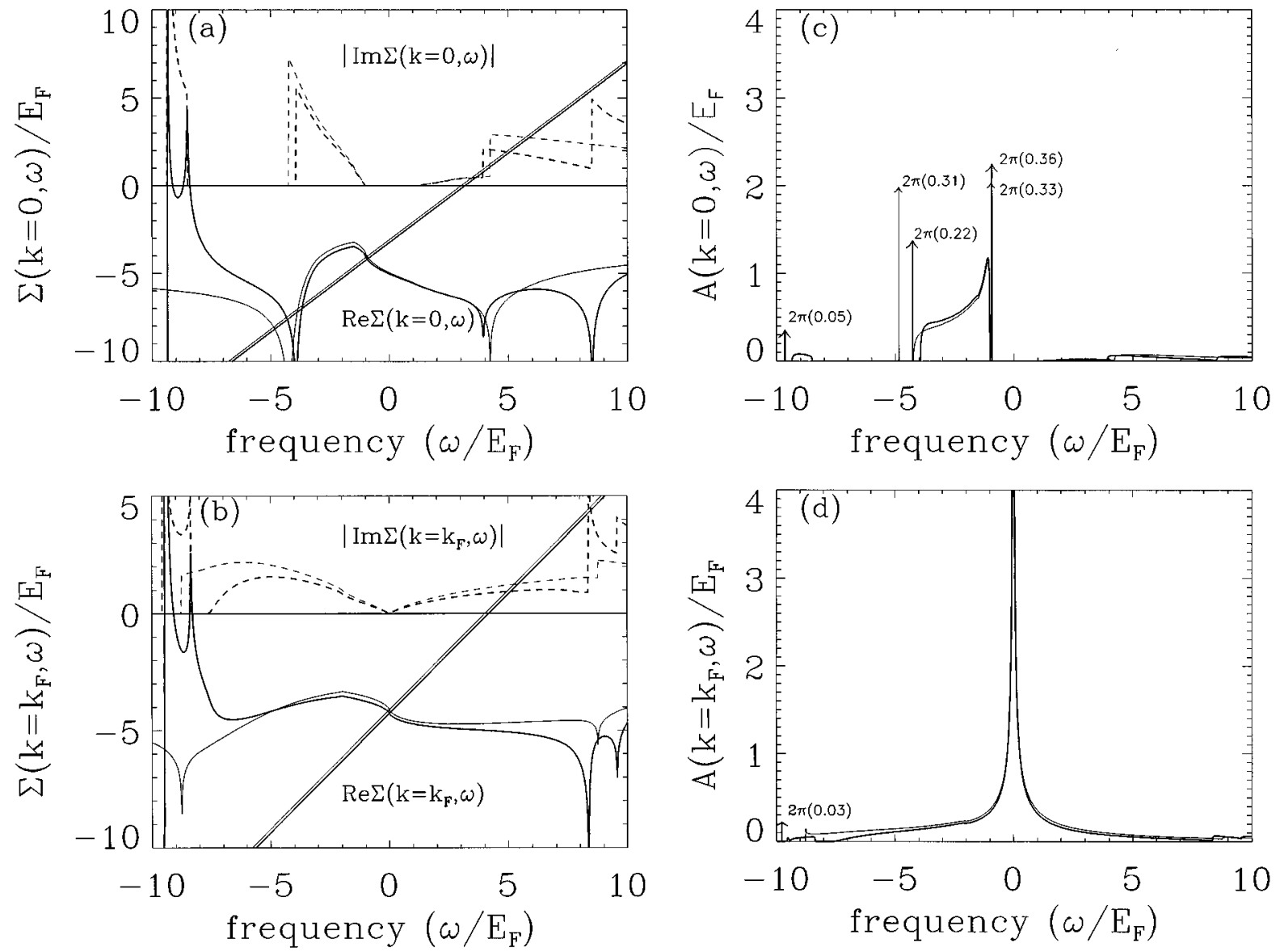

FIG. 2. Self-energy $\Sigma(k, \omega)\left[(\mathrm{a})\right.$ for $k=0$ and (b) for $\left.k=k_{F}\right]$ and spectral function $A(k, \omega)$ [(c) for $k=0$ and (d) for $\left.k=k_{F}\right]$ as functions of the frequency $\omega$ without any impurity scattering $(\gamma=0)$. Solid (dashed) curves indicate real (imaginary) parts of the self-energy. The straight lines are given by $\omega-\xi_{k}-\mu$, and their interactions with $\operatorname{Re}[\Sigma]$ indicate the solutions to Dyson's equation and correspond to a quasiparticle peak. For clarity, we plot $|\operatorname{Im} \Sigma|$. Thin (thick) lines correspond to the uncoupled (coupled) 1DEG. The vertical arrows in (c) and (d) denote $\delta$ functions in the spectral function. For $k=0$, we find at $\omega=-4.85$ and -0.96 with weights $2 \pi \times 0.31$ and $2 \pi \times 0.33$, respectively for the uncoupled system, and at $\omega=-9.65,-4.28$, and -0.96 with weights $2 \pi \times 0.05,2 \pi \times 0.22$, and $2 \pi \times 0.36$, respectively, for the coupled system. For $k=k_{F}$, we find a peak at $\omega=-9.81$ with weight $2 \pi \times 0.03$ only for the coupled system. The parameters corresponding to GaAs are used: $m=0.07 m_{\mathrm{e}}$ ( $m_{\mathrm{e}}$ is the free electron mass), $\epsilon_{0}=12.9, \epsilon_{\infty}=10.9$, and $\omega_{\mathrm{LO}}=36.8$ meV. The well width of $a=100 \AA$ and the electron gas density of $n=0.56 \times 10^{6} \mathrm{~cm}^{-1}$, which corresponds to a Fermi energy $E_{F} \approx 4.4 \mathrm{meV}$ and a dimensionless density parameter $r_{s}=4 m e^{2} / \pi k_{F} \epsilon_{0}=1.4$ with $k_{F}=\pi n / 2$, are used for this calculation.

function. The first $\delta$-function peak in $A(k, \omega)$ (with $Z=2 \pi \times 0.33$ ), which corresponds to the usual quasiparticle (i.e., a bare particle surrounded by a cloud of virtual plasmons and particle-hole excitations), is slightly shifted from the noninteracting single-particle energy $\omega=\xi(k)$. The second $\delta$-function peak (with $Z=2 \pi \times 0.31$ ) has been called a plasmaron, which is interpreted as a hole coupled to a cloud of real plasmons. For the Fermi wave vector $\left(k=k_{F}\right)$, there is only one solution to Dyson's equation at $\omega=0$ and a strong peak in $A(k, \omega)$. However, this peak is not the $\delta$-function peak, since $\operatorname{Im}\left[\Sigma\left(k_{F}, \omega\right)\right] \rightarrow \omega \sqrt{\ln \omega}$ as $\omega \rightarrow 0 .{ }^{11}$ Thus, the uncoupled 1 DEG without any impurity scattering is not a Fermi liquid even within the leading-order $G W$ approximation, ${ }^{11}$ and does not have well-defined quasiparticles. This was already reported in Ref. 11.

Since $\operatorname{Im}\left[\epsilon_{c}(q, \omega)\right]^{-1} \neq 0$ (the subscript $c$ stands for the Coulomb part; i.e., the uncoupled system) within the electron-hole continuum, the contribution to the imaginary part of the self-energy, $\operatorname{Im}[\Sigma(k, \omega)]=\operatorname{Im}\left[\Sigma_{\text {pole }}(k, \omega)\right]$, comes from both the electron-hole continuum and the collective plasmon mode. $\operatorname{Im}\left[\epsilon_{c}(q, \omega)\right]^{-1}$ can be written as

$$
\begin{aligned}
\operatorname{Im} \frac{1}{\epsilon_{c}(q, \omega)}= & \frac{-\epsilon_{2}}{\epsilon_{1}^{2}+\epsilon_{2}^{2}}\left[\theta\left(\left|\omega_{1}\right|-\omega\right) \theta\left(\omega-\left|\omega_{2}\right|\right) \theta(\omega)\right. \\
& \left.+\theta\left(-\left|\omega_{2}\right|-\omega\right) \theta\left(\omega+\left|\omega_{1}\right|\right) \theta(-\omega)\right] \\
& -\pi\left[\left.\frac{\partial \epsilon_{1}}{\partial \omega}\right|_{\omega=\omega_{\mathrm{pl}}}\right] \delta\left(\omega-\omega_{\mathrm{pl}}\right)
\end{aligned}
$$

where $\epsilon_{1}=\operatorname{Re}[\epsilon(q, \omega)]$ and $\epsilon_{2}=\operatorname{Im}[\epsilon(q, \omega)]$, and the plasmon dispersion, $\omega_{\mathrm{pl}}$, is given by ${ }^{15}$

$$
\omega_{\mathrm{pl}}(q)=\left[\frac{g(q) \omega_{1}^{2}-\omega_{2}^{2}}{g(q)-1}\right]^{1 / 2},
$$

where $g(q)=\exp \left[q \pi / m V_{c}\right], \omega_{1}=2 q+q^{2}$, and $\omega_{2}=2 q-q^{2}$. The first (second) term in Eq. (25) gives the electron-hole (plasmon) contribution. From Eq. (17), we see that the non- 
zero electron-hole contribution shows up in $\operatorname{Im}[\Sigma(k, \omega)]$ only if $\omega>1-k^{2}$ for $\omega>0$ and if $-(k+1)(k+3)$ $<\omega<-\left|1-k^{2}\right|$ for $\omega<0$. The plasmon part [the second term in Eq. (25)] contributes to $\operatorname{Im}[\Sigma(k, \omega)]$ if $\omega>\omega_{\mathrm{pl}}(1 \pm k)$ for $\omega>0$ and if $-\omega_{\mathrm{pl}}(1 \pm k)<\omega<-\left|1-k^{2}\right|$ for $\omega<0$. Thus, for $k=0, \operatorname{Im}[\Sigma(k, \omega)] \neq 0$ in the range $-\omega_{\mathrm{pl}}(1)<\omega<-1$ and $\omega>1$ [the electron-hole contribution, $\omega>1$ and $-3<\omega<-1$, and the plasmon contribution, $-\omega_{\mathrm{pl}}(1)<\omega<-1$ and $\left.\omega>\omega_{\mathrm{pl}}(1)\right]$. Since the plasmon contributions turn on at $\omega= \pm \omega_{\mathrm{pl}}(1)$ the finite discontinuities in $\operatorname{Im}[\Sigma(k, \omega)]$ take place at those points with the magnitude $\pi /\left.\left(\partial \epsilon_{1} / \partial \omega\right)\right|_{\omega= \pm \omega_{\mathrm{pl}}}$. A finite discontinuity in $\operatorname{Im}[\Sigma]$ gives rise to a logarithmic singularity in $\operatorname{Re}[\Sigma]$, which can be verified using the Kramers-Kronig relation. For $k=k_{F}, \operatorname{Im}[\Sigma]$ $\neq 0$ in the range $\omega>0$ and $-\omega_{\mathrm{pl}}(2)<\omega<0$ [the electronhole contribution, $\omega>0$ and $-6<\omega<0$, and the plasmon contribution, $\omega>\omega_{\mathrm{pl}}(2)$ and $\left.-\omega_{\mathrm{pl}}(2)<\omega<0\right]$. Turning on the plasmon contribution at $\omega= \pm \omega_{\mathrm{pl}}(2)$ induces the finite discontinuity in $\operatorname{Im}[\Sigma]$ and a logarithmic singularity in $\operatorname{Re}[\Sigma]$.

For the coupled 1DEG and $k=0$, we find three undamped solutions and two damped solutions in Dyson's equation. The first $\delta$-function peak in $A(k, \omega)$ near $\omega=0$, the quasiparticle peak, occurs at almost the same energy as the uncoupled case, but with slightly higher strength $(Z=2 \pi \times 0.36)$. The second $\delta$-function peak, the plasmaron peak, is shifted toward the Fermi energy and has much weaker strength $(Z=2 \pi \times 0.22)$ than in the uncoupled 1DEG, since the coupled plasmonlike mode has a smaller energy and weaker strength than in the uncoupled case. ${ }^{8} \mathrm{Un}-$ like the uncoupled system, we now find a third $\delta$-function peak with strength $Z=2 \pi \times 0.05$ near the frequency $\omega=-E_{F}-\omega_{\mathrm{LO}}$. This phonon satellite can be interpreted as being similar to the plasmaron, that is, a quasihole coupled to real LO phonons. For $k=k_{F}$ we find only two peaks: the quasiparticle peak at $\omega=0$ and the phonon satellite (the $\delta$-function peak with strength $Z=2 \pi \times 0.03)$. As $\omega \rightarrow 0$ the main contribution to $\operatorname{Im}[\Sigma]$ comes from a plasmon, since the phonon part has a gap at $q=0$ and the integration is performed near $q=0$. Thus, the behavior of $\operatorname{Im}[\Sigma]$ for the coupled system is the same as that for the uncoupled system and the quasiparticlelike peak near $\omega=0$ is not a strict $\delta$-function peak, which means that the coupled 1DEG with electron-phonon interaction is not a Fermi liquid either, and has no true long-lived quasiparticles.

For the coupled system, there is also a direct phonon contribution to the imaginary part of the self-energy along with the electron-phonon and plasmon contribution discussed above. $\operatorname{Im}\left[\epsilon_{t}\right]^{-1}$ can be written as

$$
\begin{aligned}
\operatorname{Im} \frac{1}{\epsilon_{t}(q, \omega)}= & \frac{-\epsilon_{2}}{\epsilon_{1}^{2}+\epsilon_{2}^{2}}\left[\theta\left(\left|\omega_{1}\right|-\omega\right) \theta\left(\omega-\left|\omega_{2}\right|\right) \theta(\omega)\right. \\
& \left.+\theta\left(-\left|\omega_{2}\right|-\omega\right) \theta\left(\omega+\left|\omega_{1}\right|\right) \theta(-\omega)\right] \\
& -\pi\left[\left.\frac{\partial \epsilon_{1}}{\partial \omega}\right|_{\omega=\omega_{\mathrm{pl}}^{(-)}}\right]^{-1} \delta\left(\omega-\omega_{\mathrm{pl}}^{(-)}\right) \\
& -\pi\left[\left.\frac{\partial \epsilon_{1}}{\partial \omega}\right|_{\omega=\omega_{\mathrm{pl}}^{(+)}}\right]^{-1} \delta\left(\omega-\omega_{\mathrm{pl}}^{(+)}\right),
\end{aligned}
$$

where $\omega_{\mathrm{pl}}^{(-)}$and $\omega_{\mathrm{pl}}^{(+)}$are the plasmonlike and the phononlike collective modes in the coupled system, respectively. ${ }^{8}$ Their behaviors in the long wavelength limit $(q \rightarrow 0)$ are given by

$$
\omega_{\mathrm{pl}}^{(+)}(q)=\omega_{\mathrm{LO}}\left[1+\frac{\omega_{\mathrm{LO}}^{2}-\omega_{\mathrm{TO}}^{2}}{\omega_{\mathrm{LO}}^{4}} 2 r_{s}|\ln (q a)| q^{2}+O\left(q^{4}\right)\right],
$$

and

$$
\omega_{\mathrm{pl}}^{(-)}(q)=\frac{\omega_{\mathrm{TO}}}{\omega_{\mathrm{LO}}} 2 q \sqrt{r_{s}|\ln (q a)|}+O\left(q^{3}\right) .
$$

The contribution to $\operatorname{Im}[\Sigma]$ from the electron-hole continuum is the same as that for the uncoupled system, but the contribution of the plasmonlike mode, $\omega_{\mathrm{pl}}^{(-)}$, is the same only as long as $\omega<\omega_{\mathrm{TO}}$, since the plasmonlike mode ceases for $\omega>\omega_{\mathrm{TO}}$ (see Ref. 8). The discontinuities in $\operatorname{Im}[\Sigma]$ induced by this mode move toward the Fermi energy $(\omega=0)$ and the strength of the step becomes smaller, since $\left|\omega_{\mathrm{pl}}\right|>\left|\omega_{\mathrm{pl}}^{(-)}\right|$. The phononlike mode contributes to $\operatorname{Im}[\Sigma]$ if the following conditions are satisfied: if $\omega>0, q^{2}-1-\omega+\omega_{\mathrm{pl}}^{(+)}=0$ with $1<q<\sqrt{\omega+1}$, and if $\omega<0, q^{2}-1-\omega-\omega_{\mathrm{pl}}^{(+)}=0$ with $0<q<1$. From these conditions we find the range in which the phononlike mode contributes to the $\operatorname{Im}[\Sigma]$ to be $\omega>\omega_{\mathrm{pl}}^{(+)}(1 \pm k)$ and $-1-\omega_{\mathrm{pl}}^{(+)}(k)<\omega<-\omega_{\mathrm{pl}}^{(+)}(1-k)$. As for the plasmon, turning on the phononlike mode produces a discontinuity in $\operatorname{Im}[\Sigma]$ and a singularity in $\operatorname{Re}[\Sigma]$. For $k=k_{F}$, since $\omega_{\mathrm{pl}}^{(+)}(1 \pm k) \geqslant \omega_{\mathrm{TO}}$, the abrupt steps in $\operatorname{Im}[\Sigma]$ for $\omega>0$ are due to the phononlike mode.

Figure 3 shows the results for the coupled 1DEG with impurity scattering effects $\left(\gamma=E_{F}\right)$. The inclusion of impurity scattering introduces collisional damping in the collective modes. Since the 1D plasmon is strongly affected by impurity scattering (i.e., they become overdamped at small $q$ ), the damping of the plasmaron (the second peak in $A$ from $\omega=0)$ peak is stronger than that for other excitations. However, the phonon part of the dynamical dielectric function is not affected by the impurity scattering. Thus, the phonon satellite (the third peak from $\omega=0$ in $A$ ) still shows a sharp peak ( almost undamped), and the abrupt steps in $\operatorname{Im}[\Sigma]$ still exist at $\omega= \pm \omega_{\mathrm{LO}}$ and $\omega=-E_{F}-\omega_{\mathrm{LO}}$. The discontinuities in $\operatorname{Re}[\Sigma]$ and the singularities in $\operatorname{Re}[\Sigma]$ induced by the plasmonlike mode are both suppressed by the impurity scattering. For $k=k_{F}$, the contribution to $\operatorname{Im}\left[\Sigma\left(k=k_{F}, \omega\right)\right]$ at $\omega=0$ mainly comes from the single-electron-hole pair excitations as in the uncoupled 1DEG, since the long wavelength plasmon is now overdamped and the phonon has a gap at a small $q$. As $\omega \rightarrow 0$, the contribution to $\operatorname{Im}[\Sigma]$ from the single-particle excitations is given by $\omega^{2}|\ln \omega|^{3}$, and $A\left(k_{F}, \omega\right) \sim|\ln (|\omega|)|^{3}+2 \pi Z_{F} \delta(\omega) .{ }^{11}$ Our direct numerical calculation shows that the weights of the $\delta$ function are $2 \pi \times 0.31$ for the uncoupled system and $2 \pi \times 0.33$ for the coupled system. Therefore, the coupled 1DEG with impurity scattering is a Fermi liquid similar to the behavior found in Ref. 11 for the uncoupled system in the presence of impurity scattering.

In Fig. 4, we show the strength $Z(k)$ of the undamped excitations for uncoupled (thin lines) and coupled (thick lines) system without impurity scattering as a function of wave vector. As the wave vector increases, the strength of 

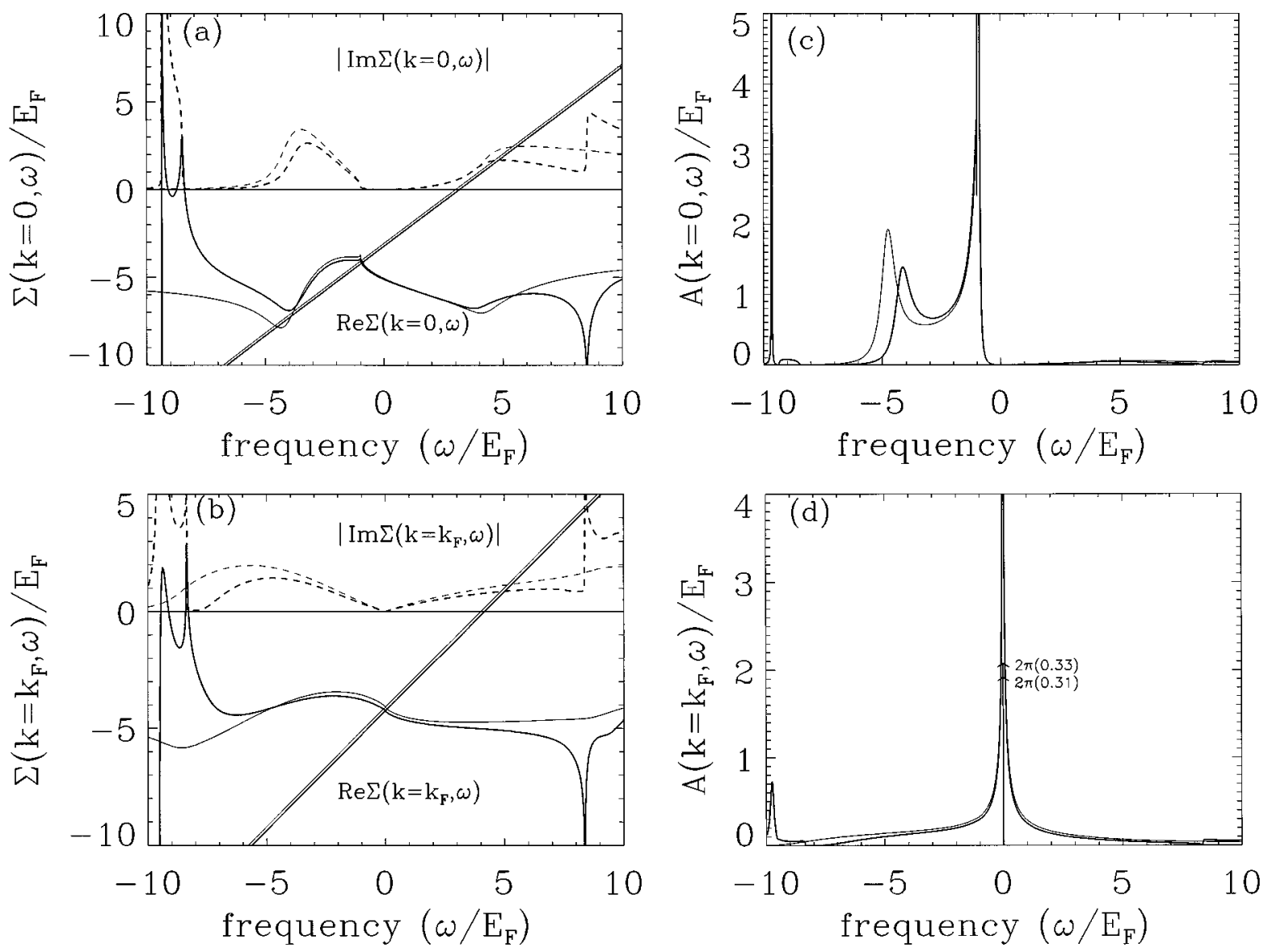

FIG. 3. Self-energy $\Sigma(k, \omega)\left[(\mathrm{a})\right.$ for $k=0$ and (b) for $\left.k=k_{F}\right]$ and spectral function $A(k, \omega)$ [(c) for $k=0$ and (d) for $\left.k=k_{F}\right]$ as functions of the frequency $\omega$ with impurity effects $\left(\gamma=E_{F}\right)$. Solid (dashed) curves indicate real (imaginary) parts of the self-energy. For clarity we plot $|\operatorname{Im} \Sigma|$. Thin (thick) lines correspond to the uncoupled (coupled) 1DEG. For $k=k_{F}$, vertical arrows in the spectral function at $\omega=0$ denote $\delta$ functions with weight $2 \pi \times 0.31$ for uncoupled system and $2 \pi \times 0.33$ for uncoupled system.

the $\delta$-function peaks for the plasmarons (dashed lines) decrease quickly and vanish at $k=0.75 k_{F}$ for the uncoupled system and at $k=0.3 k_{F}$ for the coupled system, but the strengths of the regular quasiparticle peaks (solid lines) and the phonon satellite peak (dot-dashed line) decrease slowly. On the other hand, as the wave vector approaches the Fermi momentum the strength of the quasiparticle peak decreases and vanishes at the Fermi momentum. Analytically, we can show that this behavior is logarithmic as in the uncoupled system. The most dominant term in the derivative of the real part of the self-energy comes from $\Sigma_{\text {pole }}$ as $\omega \rightarrow 0$. From Eq. (16), we have as $\omega \rightarrow 0$

$$
\begin{aligned}
\left.\frac{\partial \Sigma_{\text {pole }}}{\partial \omega}\right|_{k=k_{F}}= & \frac{1}{2 \pi} \int_{-\infty}^{\infty} d q \delta\left(\omega-\xi_{k+q}\right) v_{c}(q) \\
& \times\left[\frac{1}{\epsilon_{t}\left(q, \xi_{k+q}-\omega\right)}-1\right] \\
= & \frac{1}{2 \pi}\left[v_{c}\left(\frac{\omega}{2}\right)\left\{\frac{1}{\epsilon_{t}\left(\frac{\omega}{2}, 0\right)}-1\right\}+v_{c}(2)\right. \\
& \left.\times\left\{\frac{1}{\epsilon_{t}(2,0)}-1\right\}\right] .
\end{aligned}
$$

Thus, as $\omega \rightarrow 0$ the first term shows a logarithmic singularity,

$$
\left.\frac{\partial \Sigma_{\text {pole }}}{\partial \omega}\right|_{k=k_{F}} \sim-(\ln |\omega|+1) .
$$

This demonstrates that even within our leading-order approximation, the coupled system without impurity scattering is not a Fermi liquid, since the renormalization constant at $k=k_{F}$ vanishes (i.e., the momentum distribution is continuous at the Fermi momentum).

Figure 5(a) shows the quasiparticle damping rate $\Gamma(k)$ for both the coupled system (thick lines) and the uncoupled system (thin lines) with the parameters $a=100 \AA$ and $n=0.56 \times 10^{6} \mathrm{~cm}^{-1}$. [The inset in Fig. 5(a) shows the plasmon-phonon mode coupling.] The corresponding inelastic mean free paths $l_{K}$ are shown in Fig. 5(b). For the uncoupled 1DEG, the quasiparticle scatters by plasmon emission, which corresponds to the sharp threshold in the thin solid line shown in Fig. 5(a). There is no 1D single-particle electron-electron scattering below a threshold critical wave vector, because the conservation of energy and momentum restricts electron-electron scattering to an exchange of particles. In 2D and 3D systems, there is scattering allowed below the threshold critical wave vector due to the excitation of electron-hole pairs. For the coupled 1DEG, the quasipar- 


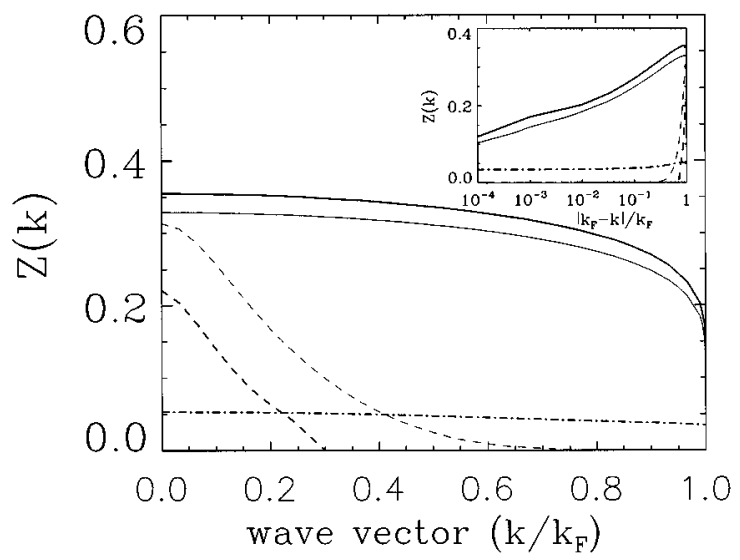

FIG. 4. Strength of the undamped excitations [the quasiparticle renormalization factor $Z(k)$ ] for uncoupled (thin lines) and coupled (thick lines) system without impurity scattering as a function of wave vector. Solid lines (dashed lines) denote quasiparticle peaks (plasmarons) and dot-dashed line a phonon satellite peak. Inset shows the logarithmic approach of the renormalization factor to zero as $k \rightarrow k_{F}$.

ticle decays via the emission of coupled plasmon-phonon $\operatorname{mode}^{20}\left[\omega_{-}\right.$and $\omega_{+}$in the inset of Fig. 5(a) $]$, which corresponds to the two peaks in the thick solid line of Fig. 5(a). The first step corresponds to the $\omega_{-}$-emission threshold and is located below the $k_{c}$ of the uncoupled 1DEG, because $\omega_{-}<\omega_{0}$. The second step corresponds to the $\omega_{+}$-emission threshold and occurs at a wave vector larger than $k=\sqrt{\omega_{\mathrm{LO}}+1}$, because $\omega_{+}>\omega_{\mathrm{LO}}$. When only Fröhlich interaction in the $1 \mathrm{D}$ quantum wire is considered, ${ }^{21}$ the LOphonon emission threshold has been predicted at the fixed critical wave vector $k=\sqrt{\omega_{\mathrm{LO}}+1}$. But in our coupled model the locations of $\omega_{+}$-emission threshold depend on the density of the electron as in the plasmon emission threshold. The thin lines in Fig. 5(a) show the damping rate with the impurity scattering effects $\left(\gamma=E_{F}\right)$. Since the plasmon line is broadened by impurity scattering, there are inelastic scattering events below the critical wave vectors. The breaking of translational invariance by impurities, furthermore, relaxes momentum conservation and permits inelastic scattering via single-particle excitation (except at the Fermi surface where the quasiparticle is always undamped). Thus, the nature of the sharp threshold in our doped system is totally different from that discussed in Ref. 21, which considers undoped quantum wires. The sharp divergent thresholds obtained in Ref. 21 result from the divergence in the density of states at the bottom of the band of one-dimensional systems, and therefore in doped samples, when the bottom of the band is no longer accessible, these sharp thresholds do not occur. In our theory, the divergences are due to the divergence in the joint density of states at the coupled plasmon-phonon emission threshold, and they occur at any doping.

Based on these results, we propose an interesting device principle of a one-dimensional quantum wire, which was originally suggested by Hu and Das Sarma for the uncoupled system. ${ }^{22}$ The device principle that we propose is based on the many-body properties discussed in this paper, which are peculiar to an interacting one-dimensional Fermi system. It may be possible to fabricate a hot-electron transistor device
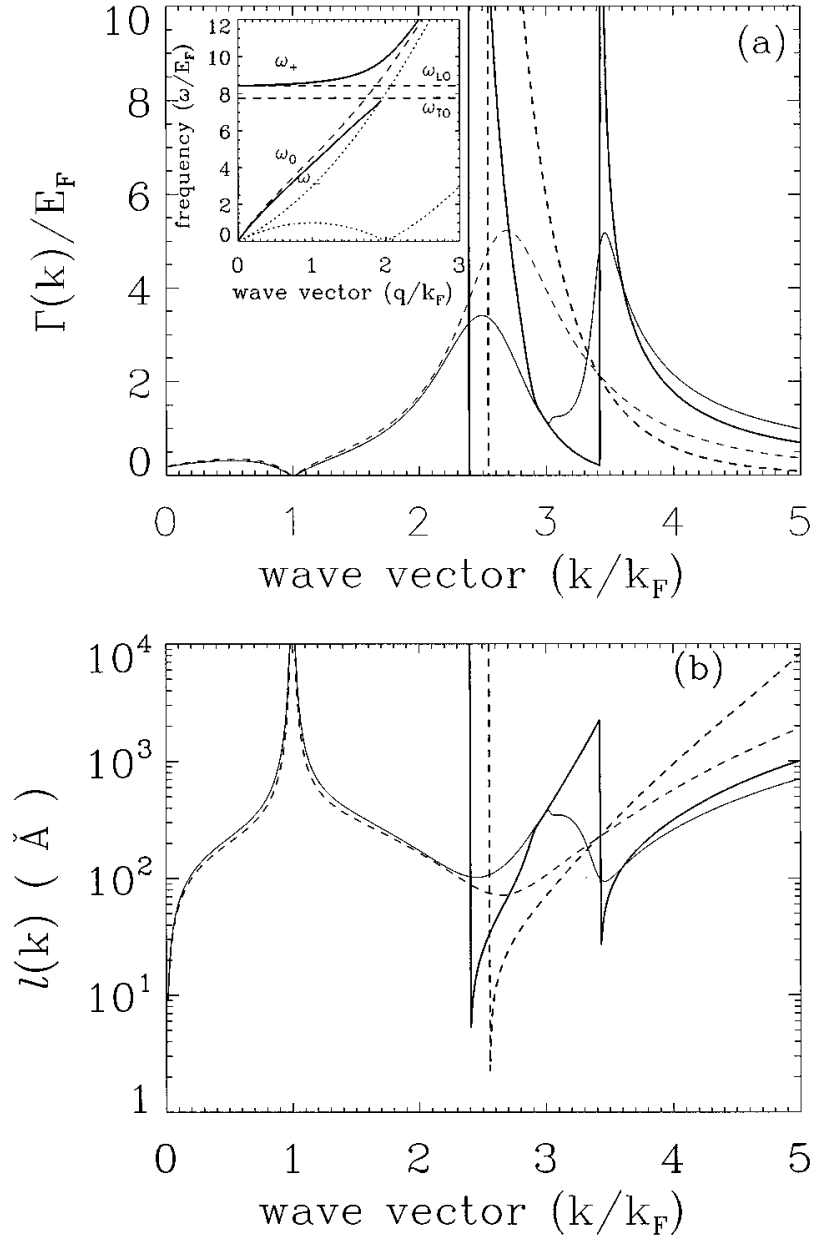

FIG. 5. (a) Damping rate $\Gamma(k)$ and (b) the corresponding mean free path $l(k)=v(k) / 2 \Gamma(k)$ as a function of $k$. Solid (dashed) lines indicate the coupled (uncoupled) system and thick (thin) lines for $\gamma=0\left(\gamma=E_{F}\right)$. The inset in (a) shows the plasmon-phonon mode coupling.

with a large and a sudden onset of negative differential resistance (NDR) associated with sharp changes in the inelastic mean free path due to electron-coupled mode scatterings of the injected electrons at $T=0$. This sudden onset of NDR could be exploited to produce a transistor, while the NDR itself suggests that this device might be used as an oscillator. The device principle which we propose may be experimentally observed in the quasi-one-dimensional version of the tunneling hot-electron transistor amplifier (THETA) (Refs. 23 and 24) or the resonant tunneling hot-electron transistors (RHET's), ${ }^{25}$ which have been fabricated successfully in GaAs-based electron gas systems in three and two dimensions.

In a two-dimensional electron gas, the dominant inelastic scattering of hot electrons for low carrier concentrations is due to the single-particle excitation, emission of plasmons, and emission of LO phonons, and the scattering due to coupled modes dominates only in the higher electron densities. ${ }^{7}$ In order to emit LO phonons, a hot electron needs to gain an excess energy equal to at least the LO-phonon energy $\left(\approx 36 \mathrm{meV}\right.$ in GaAs). ${ }^{24}$ Unlike the two-dimensional electron gas, in 1DEG the coupling is found to be strong for all densities ${ }^{8}$ and the single-particle excitations are severely 
suppressed by the phase space restriction, due to $1 \mathrm{D}$ energymomentum conservation. Thus, the main scattering mechanism for the $1 \mathrm{D}$ electrons in the one-dimensional THETA devices arises from the coupled plasmon-LO phonon mode. Due to the strong mode coupling between plasmons and LO phonons the emission of the $\omega_{+}$mode requires that the energy of a hot electron be higher than the LO-phonon energy. When the excess energy of electrons is lower than LOphonon energy only the $\omega_{-}$mode contributes to the scattering. This effects should be directly observable experimentally.

In higher-dimensional electron systems there are also coupled-mode thresholds at the onsets of coupled-mode emissions in the high density limit. The effect in higher dimensions, however, is not dramatic because the ideal mean free path does not change from being infinite below the threshold to zero above (as it does in one dimension) because the single-particle scattering contributes in higher dimensions in contrast to one dimension. Thus, our proposed NDR effect in quantum wires is a specific one-dimensional manybody property which should be experimentally observable.

\section{SUMMARY AND CONCLUSION}

In this paper, we have calculated the quasiparticle selfenergy, the spectral function, and the damping rate within the leading-order dynamical screening approximation by treating electron-electron and electron-phonon interactions on an equal footing both with and without impurity scattering effects. Our many-body theory includes the important physical effects of dynamical screening, phonon self-energy correction, plasmon-phonon mode coupling, Fermi statistics, and Landau damping. Even though the problem is treated within the $G W$ framework of the leading-order effective interaction approximation (i.e., RPA screening and neglect of vertex corrections), our results should be quite valid in GaAs-based 1DEG. This is because GaAs has a very weak Fröhlich coupling, which justifies the neglect of the electron-phonon ver- tex corrections, and its low effective mass and the large dielectric constants gives a small effective $r_{s}$ parameter (and in this limit generally the RPA is valid), which makes direct electron-electron interaction also weak in the perturbative sense.

The quasiparticle properties at $\omega \approx \xi_{k}$ are affected only by low-energy processes and hence are not changed much by the inclusion of the optic-phonon coupling, because the optic-phonon modes have a gap to excitation. The opticphonon coupling does, however, shift some of the spectral weight to an extra undamped excitation at $\omega \approx-\left(E_{F}+\omega_{\mathrm{LO}}\right)$, which is the phonon analogy to the plasmaron excitation, and it shifts the plasmaron energy up slightly, due to level repulsion between the plasmaron and the phonon excitation. As in the uncoupled system, inclusion of impurity scattering tends to smear out the above features.

The properties at the Fermi surface, such as the impuritydriven restoration of the discontinuity in the distribution function, are virtually the same for the coupled and uncoupled systems, again because of the gap of the opticphonon mode. At higher energies, the coupled system has an additional phononlike mode $\omega_{+}$which can scatter electrons. This leads to another divergence in the scattering rate $\Gamma(k)$ (i.e., in addition to the one from plasmon emission). This sudden onset of the divergence in the scattering rate can, in principle, be used in a one dimensional THETA or RHETtype device leading to the possibility of a large negative differential resistance. This peculiarly 1D characteristic has potential applications such as switching devices and oscillators. Note that this purely many-body divergence occurs only when one couples the electron-electron and electron-phonon interactions together; no such divergence is obtained for calculations in doped systems using only the Fröhlich interaction.

\section{ACKNOWLEDGMENTS}

This work is supported by the U.S.- ARO and U.S.- ONR.
${ }^{1}$ For reviews see, e.g., Nanostructure Physics and Fabrication, edited by M. A. Reed and W. P. Kirk (Academic, New York, 1989); Nanostructures and Mesoscopic Systems, edited by W. P. Kirk and M. A. Reed (Academic, New York, 1992).

${ }^{2}$ R. Cingolani, H. Lage, L. Tapfer, H. Kalt, D. Heitmann, and K. Ploog, Phys. Rev. Lett. 67, 891 (1991); A. S. Plaut, H. Lage, P. Grambow, D. Heitman, K. von Klitzing, and K. Ploog, ibid. 67, 1642 (1991); J. S. Wiener, J. M. Calleja, B. S. Dennis, L. N. Pfeiffer, and K. W. West, ibid. 67, 3298 (1991); J. M. Calleja, A. R. Goñi, B. S. Dennis, J. S. Weiner, A. Pinczuk, S. SchmidttRink, L. N. Pfeiffer, K. W. West, J. R. Muller, and A. E. Ruckenstein, Solid State Commun. 79, 911 (1991).

${ }^{3}$ M. Tsuchiya, J. M. Gaines, R. H. Yam, R. J. Simes, P. O. Holtz, L. A. Colden, and P. M. Petroff, Phys. Rev. Lett. 62, 466 (1989); R. Nötzel, N. N. Ledentsov, L. Däweritz, K. Ploog, and M. Hohenstein, Phys. Rev. B 45, 3507 (1992).

${ }^{4}$ E. Kapon, D. M. Hwang, and R. Bhat, Phys. Rev. Lett. 63, 430 (1989).

${ }^{5}$ K. Kash, B. P. Van der Gaag, D. D. Mahoney, A. S. Gozdz, L. T.
Florez, J. P. Harbison, and M. D. Sturge, Phys. Rev. Lett. 67, 1326 (1991).

${ }^{6}$ L. Pfeiffer, K. W. West, H. L. Störmer, J. P. Eisenstein, K. W. Baldwin, D. Gershoni, and J. Spector, Appl. Phys. Lett. 56, 1697 (1990); D. Gershoni, J. S. Weiner, S. N. G. Chu, G. A. Baraff, J. M. Vandenberg, L. N. Pfeiffer, K. W. West, R. A. Logan, and Tanbun-Ek, Phys. Rev. Lett. 65, 1631 (1990); H. L. Stormer, L. N. Pfeiffer, K. W. Baldwin, K. W. West, and J. Spector, Appl. Phys. Lett. 58, 726 (1991); A. R. Goñi, L. N. Pfeiffer, K. W. West, A. Pinzuk, H. U. Baranger, and H. L. Stormer, ibid. 61, 1956 (1992).

${ }^{7}$ R. Jalabert and S. Das Sarma, Phys. Rev. B 40, 9723 (1989).

${ }^{8}$ E. H. Hwang and S. Das Sarma, Phys. Rev. B 52, R8668 (1995).

${ }^{9}$ For review see, e.g., The Many-Body Problem, edited by Danial C. Mattis (World Scientific, Singapore, 1993); Bosonization, edited by Michael Stone (World Scientific, Singapore, 1994).

${ }^{10}$ G. Mahan, Many Particle Physics (Plenum, New York, 1988).

${ }^{11}$ Ben Yu-Kuang Hu and S. Das Sarma, Phys. Rev. Lett. 68, 1750 (1992); Phys. Rev. B 48, 5469 (1993). 
${ }^{12}$ L. Hedin and S. Lundqvist, in Solid State Physics: Advances in Research and Applications, edited by H. Ehrenreich, F. Seitz, and D. Turnbull (Academic, New York, 1969), Vol. 23.

${ }^{13}$ T. Ando, A. B. Fowler, and F. Stern, Rev. Mod. Phys. 54, 437 (1982), and references therein.

${ }^{14}$ Q. P. Li, S. Das Sarma, and R. Joynt, Phys. Rev. B 45, 13713 (1992), and references therein.

${ }^{15}$ Q. P. Li and S. Das Sarma, Phys. Rev. B 43, 11768 (1991), and references therein.

${ }^{16}$ Note that Eq. (2.5) in Ref. 11 contains typographical errors which are corrected here.

${ }^{17}$ N. D. Mermin, Phys. Rev. B 1, 2362 (1970).

${ }^{18}$ L. Hedin, Phys. Rev. 139, A796 (1965).

${ }^{19}$ J. J. Quinn and R. A. Ferrell, Phys. Rev. 112, 812 (1958).
${ }^{20}$ M. E. Kim, A. Das, and S. D. Senturia, Phys. Rev. B 18, 6890 (1978).

${ }^{21}$ F. A. Riddoch and B. K. Ridley, Surf. Sci. 142, 260 (1984); E. S. Hellman and J. S. Harris, ibid. 174, 459 (1986).

${ }^{22}$ Ben Yu-Kuang Hu and S. Das Sarma, Appl. Phys. Lett. 61, 1208 (1992).

${ }^{23}$ A. F. Levi et al., Phys. Rev. Lett. 55, 2071 (1985); M. Heiblum, M. I. Nathan, D. C. Thomas, and C. M. Knoedler, ibid. 55, 2200 (1985); M. Heiblum et al., ibid. 58, 816 (1987).

${ }^{24}$ M. Heiblum, D. Galbi, and M. Weckwerth, Phys. Rev. Lett. 62, 1057 (1989); U. Sivan, M. Heiblum, and C. P. Umbach, ibid. 63, 992 (1989).

${ }^{25}$ N. Yokoyama et al., in Hot Carriers in Semiconductor Nanostructures, edited by J. Shah (Academic Press, San Diego, 1992). 